\title{
Un puente para el encuentro de la arqueología y la bioantropología: el extrañamiento de los modos de vida*
}

\author{
Florence Constantinescu
}

\section{INTRODUCCIÓN}

Tradicionalmente, la arqueología estudia las sociedades del pasado por medio de las evidencias que quedaron de las antiguas actividades humanas. Su fin es reconstruir la conducta de las sociedades extintas, tratando de abarcar el rango completo de actividades humanas realizadas por cada cultura.

El objeto de estudio de la arqueología - el registro arqueológico- está compuesto por tres tipos básicos de datos: los artefactos, los ecofactos y los rasgos, que se articulan en el contexto particular de cada sitio arqueológico. Los sitios se distinguen según las funciones que se piensa cumplieron en el pasado, reflejando los distintos tipos de actividades realizadas por las diversas culturas: sitios habitacionales, sitios de matanza, centros de intercambio, centros ceremoniales, áreas de entierro...

En el caso específico de los sitios de enterratorio o cementerio, los restos óseos humanos recuperados son enviados a especialistas - los bioantropólogos- para su estudio, los que entregan sus resultados al arqueólogo que incorpora estos datos a sus investigaciones. La separación entre arqueología y bioantropología se hace evidente: esta última es un campo distinto de estudio, con su propio objeto - los organismos humanos- de manera que dentro del registro arqueológico la calidad de 'esqueleto humano' nunca ha sido definida, excluyéndosela de los tres tipos de datos básicos en arqueología.

Sin embargo, ambos campos de estudio se traslapan y los estudios que han adoptado enfoques interdisciplinarios en su investigación, han aportado los resultados más fructíferos y significativos. La interdisciplinariedad ha permi-

* Artículo proveniente del proyecto FONDECYT N 1950175. 
tido flexibilizar la reflexión arqueológica, por lo que el ampliar esta flexibilidad de reflexión con el fin de mejorar la comprensión interdisciplinaria, supone utilizar el método del 'extrañamiento' (Wallner). Ello equivale a tomar el cuerpo de proposiciones y reglas de actuación que conforman una teoría y colocarla en un contexto determinado, extraño originalmente a esa teoría. De este modo, se renuncia a la ficción de que sólo por medio de determinados métodos es posible acercarse al conocimiento verdadero, pues el 'exirañamiento' permite crear nuevos contextos que se pueden intercambiar. Surgen así visiones de estructuras que antes no se daban.

En este sentido, se postula una visión integrativa de la ciencia, visión que se ha venido aplicando con una fuerza cada vez mayor en arqueología. Borrero critica el que se haya:

"abogado que las excavaciones son las bases para construir una arqueología. Esta es una posición ingenua que lleva a interpretar los materiales arqueológicos como si reflejaran directamente las actividades del pasado" (op. cit.: 164).

"No se trata de estudiar el registro de superficie prestando poca atención al material estratificado, sino de utilizar todo el registro... Reconociendo que el registro arqueológico es un continuum con variaciones en densidad (Ebert y Kohler 1988), se abre un panorama un poco más completo acerca del uso humano del espacio (Belardi 1991, Belardi y Franco 1991, Borrero et al. 1990, 1991, Goñi 1991)" (op. cit.: 162).

De esta manera, enfatiza la importancia de estudios interdisciplinarios en arqueología tales como:

"la exploración de la interacción entre ecosistemas y poblaciones humanas desde el punto de vista de los asentamientos y su distribución temporal (Goñi 1988), osteológico (Constantinescu y Aspillaga 1991, Guichón et al. 1991), las potenciales barreras geográficas (Nacuzzi 1987, Borrero 1991, Cocilovo y Guichón 1991), esquemas de poblamiento (Prieto 1988, Borrero 1989), o la distribución de los recursos (Borrero 1985, Prieto 1988, Lanata 1990, Mena 1991)" (op. cit.: 163).

Para Borrero, el integrar los datos arqueológicos y bioantropológicos dentro de una interpretación conjunta es prácticamente imperativo:

"nuestro primer objetivo científico es explicar la formación del registro arqueológico (;en superficie o estratificado!), para poder aspirar a interpretarlo. Al plantear la arqueología como el estudio del registro arqueológico, estamos incluyendo los restos humanos" (op. cit.: 164).

Es esta propuesta de Borrero la que aquí se rescata, proponiendo un nuevo enfoque para trabajar conjuntamente los datos arqueológicos y bioantropológicos, extrañando la reconstrucción de 'modos de vida'. 


\section{Modos de vida: una nueva definición para un antiguo concepto}

Reconstruir la conducta de las sociedades pasadas es la meta tanto de la arqueología como de la bioantropología, aunque ambas consideran distintos tipos de evidencia y medios para lograrlo. Sin embargo puede establecerse un puente entre ambas especialidades, trabajando el concepto de 'modo de vida' y extrañando los supuestos bioantropológicos que definen su reconstrucción hacia el campo de la arqueología. Pero el empleo del concepto 'modo de vida' resulta delicado, pues aunque no existen palabras que describan mejor a lo que apela, ha sido definido de distintas maneras que en cierta forma terminan siendo prácticamente incompatibles.

En bioantropología se entiende 'modo de vida' de manera literal: es aquello que los miembros de un grupo humano determinado, pertenecientes a una cultura determinada hacen en su vida cotidiana. La arqueología por su parte, propone dos acepciones: a) se asocia sistemáticamente a un enfoque conservador, que lo define como el estilo de vida de los cazadores-recolectores clásicos (Lee \& De Vore), o menos frecuentemente; b) se toma este término de la geografía tal como Vidal de la Blanche lo utilizó para describir a los crianceros y campesinos rurales de Francia (Thomas, com. pers.)

En el presente artículo se propone una nueva definición para este concepto, que une ambas acepciones arqueológicas ampliándolas hacia las demás sociedades humanas y operacionaliza la definición bioantropológica desde la arqueología. De manera que se entiende 'modo de vida' como los patrones conductuales definidos por una cultura particular, con que sus miembros deben operar en un ambiente físico determinado ${ }^{1}$. Por consiguiente, la cultura se refleja también en el organismo humano. El reconstruir el 'modo de vida' de una sociedad pasada implica por tanto deducir la conducta humana definiendo los patrones de actividad desarrollados por el grupo bajo estudio, principalmente mediante las patologías morfofuncionales que estas actividades causaron específicamente en sus esqueletos. Deben además analizarse la dieta, las distancias biológicas y la demografía para obtener un cuadro general de las actividades practicadas por el grupo. Una vez obtenidos los patrones de actividad, éstos se contrastarán con los resultados obtenidos en los estudios del contexto arqueológico y de la información etnohistórica y etnográfica (en el caso de los sitios tardíos e históricos). La integración de los datos bioantropológicos, arqueológicos y etnohistóricos/etnográficos —cuando correspon-

\footnotetext{
'Esta definición no propone que el 'modo de vida' tenga por finalidad la adaptación del grupo al medioambienle, pues:

a) si bien el medioambiente es importante, una cultura tiene ante determinadas situaciones, opciones isocrésticas de solución (Sackett) y, la selección sistemática y recurrente de una de ellas no está determinada por el medio;

b) el concepto 'adaptación’ es difícil de manejar pues no hay una definición de lo que adaptación significa para una cultura, por lo que en arqueología no existe un referente claro para determinar esta situación.
} 
da-- permitirá la reconstrucción interdisciplinaria del 'modo de vida' pasado de una población ${ }^{2}$.

\section{Ecología Cultural: el enfoque teórico más cercano a la reconstrucción de 'modos de vida'}

Si se considera que para reconstruir un 'modo de vida' pasado, lo ideal es: a) disponer de esqueletos en un estado de conservación tal que permita el estudio de las patologías morfofuncionales que presentan; b) conocer el contexto arqueológico de los esqueletos, y c) contar con relatos de cronistas que hayan descrito la población bajo estudio, debe antes que nada buscarse un marco teórico arqueológico adecuado para lograr interpretar la información recabada proveniente de las tres líneas de investigación anteriores. Hasta el momento, la investigación arqueológica no ha desarrollado en profundidad este tipo de estudios, por lo que tampoco ha sido propuesto un marco teórico para éstos.

Aparentemente, el enfoque arqueológico más cercano a este tipo de investigación sería la ecología cultural que propone el estudio del proceso adaptativo por medio del cual naturaleza y sociedad, junto con un número impredecible de rasgos de la cultura, son afectadas por los ajustes básicos a través de los cuales el hombre utiliza el medio en que vive. Postula que analizando empíricamente el núcleo de rasgos culturales que está principalmente relacionado con las actividades de subsistencia y la organización económica, es posible conocer estos ajustes y otorgar su justo valor a la incidencia del medio sobre el comportamiento del grupo humano.

Es Steward, quien en 1955 propone este nuevo enfoque para la arqueología, herramienta metodológica que sirve para comprobar como la adaptación de una cultura a su entorno - la interacción que se establece entre el hombre y el medioambiente - puede ocasionar cambios de estado en las sociedades.

Desde ese momento, la propuesta de Steward fue desarrollada por distintos investigadores en sus diversos ámbitos de aplicación, trabajo que puede sintetizarse en la evaluación que de este enfoque hacen Sharer \& Ashmore en 1979. Tales autores señalan que la ecología cultural provee a la arqueología de modelos sistémicos y comprehensivos de interacción entre cultura y medioambiente, considerando que ésta interactúa con tres subsistemas complejos que componen el sistema ambiental: a) el hábitat o territorio físico; b) el bioma o medio biológico, y c) el medio cultural compuesto por los grupos humanos adyacentes. Para cualquier sociedad determinada, la suma de las interacciones específicas dentro de un sistema ecológico-cultural describe la naturaleza de la adaptación cultural de la sociedad. Cada sociedad se adapta a su entorno,

\footnotetext{
2Si bien esta propuesta está planteada pensando en poblaciones pasadas, su aplicación en estudios de poblaciones actuales es perfectamente posibie.
} 
en primer lugar mediante su sistema tecnológico, pero secundariamente a través de sus sistemas social e ideal.

Sharer \& Ashmore (op. cit.) plantean que el set de interacciones en este complejo sistema es difícil de estudiar de una vez, por lo que el arqueólogo debe aislar uno o más subsistemas directamente implicados en la adaptación cultural. Lo habitual es trabajar con la tecnología para comprender los procesos adaptativos pues además, por lo general, es el registro más completo. Sin embargo, es importante saber que aunque gran parte de los cambios se originen en el subsistema tecnológico de la cultura, dichos cambios pueden ocurrir en cualquier parte del sistema ecológico-cultural.

Frente a este punto se debe hacer referencia a D. Clarke quien en 1978, hace énfasis en que es necesario un modelo general para los procesos culturales, porque los sistemas de relación estudiados por los arqueólogos son manifestaciones especiales de unos sistemas mucho más amplios que existieron en otros tiempos.

Clarke (op. cit.) señala que arqueólogos y antropólogos trabajan esencialmente con datos culturales relacionados con artefactos o actividades, razón por la cual esos aspectos tienden a adquirir un lugar preponderante en los sistemas culturales. Se sabe no obstante, que cada atributo de un artefacto equivale a una acción fósil, y cada artefacto a una secuencia de acciones o actividades petrificadas, y que un conjunto de artefactos se parangona con una pauta completa de comportamiento.

Sharer \& Ashmore (op. cit.) destacan que en el enfoque ecológico-cultural, como analogía a la adaptación biológica, muchos arqueólogos miden la efectividad de la adaptación cultural por medio de la tasa de crecimiento de población y el tamaño resultante de esta última. En este sentido, ambos factores son una respuesta mensurable de la adaptación al sistema ecológicocultural total. En relación al crecimiento de la población, éste se explica por la presencia de uno o más mecanismos de feed-back positivo (cambio tecnológico, mayor eficiencia de producción y almacenaje, acomodación de la población). Sin embargo la población también se puede mantener en equilibrio dinámico por medio de un feed-back negativo (control de la población - control de los nacimientos, infanticidio, guerra-, migración y fisión social). También contribuyen a este fenómeno algunos mecanismos del entorno, como las hambrunas periódicas y patologías endémicas.

Clarke (op. cit.) por su parte, postula que básicamente es posible reducir artefactos y actividades a un comportamiento, compuesto por acciones de los generadores del sistema. Los atributos básicos de esos sistemas culturales no son actividades, artefactos o creencias arbitrarias, sino "información' que controla y regula esas tres expresiones derivadas de la tradición cultural. El ritmo continuo de secuencias de actividad repetidas día tras día. mes tras mes, estación tras estación y año tras año, genera un código distintivo de información idiosincrática respecto al sistema cultural en 
cuestión. Las acciones individuales varían con irregularidad, pero, por encima de todo, prosigue el ritmo continuo y redundante de actividad reglada, cambiando lenta pero seguramente a escala de la vida del individuo: una intimación rítmica de información de supervivencia y de interferencias idiosincráticas comprensibles, que asegura la supervivencia de los jóvenes en el seno del grupo y la del grupo en su medio cambiante con un mínimo de ruptura.

Si bien, la ecología cultural es un marco teórico desde el cual se han seguido desarrollando principalmente distintos modelos para interpretar el registro arqueológico —estudio de patrones de asentamiento y de subsistencia mediante los conceptos de área de cobertura de recursos y de estrategias óptimas de forrajeo, sólo por nombrar dos de los más exitosos--, es difícil abarcar desde aquí lo que se pretende definir como 'modo de vida' para la arqueología.

Está claro que los humanos dejan un registro de sus actividades, aunque fragmentario e incompleto, en los restos de su cultura material. La función de una aguja es fácilmente comprensible y su presencia en un sitio sugiere su uso dentro de una gama específica de actividades. Por lo general, se puede ir más allá y discutir actividades que no están directamente relacionadas con los objetos en cuestión, sino que meramente implicadas por su presencia. Lo anterior se debe a que muchas actividades relacionadas a los objetos son transculturales, mientras que otras son más específicas.

Mas los humanos también dejan un registro de sus actividades en sus propios restos: el "uso y desgaste" de los tejidos, que queda marcado sin proponérselo, durante la realización y el transcurso las actividades diarias. Al tratar de interpretar este registro, el bioantropólogo se enfrenta a ciertos problemas que también enfrenta el arqueólogo. Ambos deben preguntarse si lo que ven, es producto de la actividad humana o producto de la naturaleza. En los restos esqueletales humanos los cambios pueden ser producidos por a) elementos químicos del aire o la tierra de la tumba; b) presión ejercida por rocas o por la misma tierra sobre los huesos; c) actividades de organismos vivientes, y d) otros factores peculiares del lugar de sepultación.

Ambos especialistas también interpretan la conducta a partir de los objetos, debiendo considerar una amplia gama de elementos contextuales que pueden ser más importantes que los objetos mismos. En cierta medida el bioantropólogo tiene la tarea más difícil, porque las relaciones entre sus objetos - la evidencia de patologías en el esqueleto-y las actividades, son mucho menos directas que aquellas del arqueólogo. Mientras que los arqueólogos han estado trabajando este problema durante largo tiempo, los intentos de ver los restos osteológicos en términos de conducta son comparativamente recientes y escasos. De hecho, muchas relaciones actividad-patología son aún poco conocidas, incluso en su contexto clínico.

Sin embargo, una ventaja de trabajar con esqueletos, es que representan 
nuestros actos básicos del pasado. Como tales, son útiles en el registro de los eventos del pasado y también pueden ser útiles en generar hipótesis relevantes para el presente.

Se ha propuesto que 'modo de vida' sea para la arqueología vida aquellos patrones conductuales definidos por una cultura particular, con que sus miembros deben operar en un ambiente físico determinado. Así, la definición de Vidal de la Blanche no corresponde aquí. Y de acuerdo a lo que se puede desprender de la ecología cultural, los 'modos de vida' deberían reducirse a la expresión concreta y singular de los modos de producción, modelo teórico que deja fuera de registro aquellas actividades que no son actividades de subsistencia.

Resulta básico entonces redefinir el enfoque teórico con que se debe trabajar para interpretar conjunta y adecuadamente los distintos tipos de registros. La ecología cultural y los enfoques posteriores que de ella se derivan, no proporcionan los elementos necesarios para trabajar el problema planteado. Se propone por tanto integrar - 'extrañar'- a los anteriores supuestos arqueológicos, el marco teórico proveniente de la bioantropología que, desarrollando en paralelo dos líneas de trabajo complementarias - la paleodemografía y la paleopatología - proporcionará la base para las investigaciones en torno a la reconstrucción de los 'modos de vida'.

\section{Paleodemografía y Paleopatología: los enfoques por 'extrañar'}

Hace aproximadamente 25 años comenzó a desarrollarse una nueva línea de investigación en el ámbito de la Antropología Física, que es el de la reconstrucción de los estilos de vida, principalmente con miras a colaborar en la identificación forense y a aportar una mayor cantidad de datos de interés en la investigación arqueológica acerca de poblaciones extintas.

\section{Paleodemografía}

Una de las metas de la bioantropología es la reconstrucción de los "modos de vida' pasados, incluyendo las actividades diarias o acostumbradas de los pueblos prehistóricos. Puesto que para desarrollar esta perspectiva, se utilizan los restos esqueletales provenientes de cementerios prehistóricos, tanto la paleopatología como la paleodemografía presuponen que existen relaciones directas entre las estadísticas calculadas a partir de las muestras esqueletales y el estado de salud de las poblaciones pasadas que dieron origen a esas muestras.

Esta interpretación trabaja con el supuesto implícito de que dentro de un cementerio, los muertos son (a lo menos en su promedio) razonablemente representativos de las poblaciones vivas que los produjeron y por tanto, que los cambios en los conjuntos esqueletales reflejan cambios reales en las poblaciones que alguna vez vivieron. 
Para Cohen (1994), si los individuos de una población estuvieran bajo el mismo riesgo de morir por todas las causas (o si todas las causas de muerte fueran estrictamente accidentales), se esperaría que un cementerio fuera por lo general una muestra representativa y casual del grupo existente. Cohen propone entonces que, excepto bajo condiciones muy extremas, el grupo de muertos real de una población en cualquier año será por lo general una fiel representación de la población existente, porque la naturaleza de las muertes es casual y no selectiva.

Sin embargo, Cohen reconoce que tal vez la mayoría de las muertes humanas están débilmente relacionadas con las enfermedades crónicas que muestran los esqueletos humanos, - o que esas patologías sólo constituyen un pequeño porcentaje de las enfermedades que contribuyen a las posibilidades de la mortalidad- y los esqueletos pueden ser una muestra relativamente casual con respecto a la patología esqueletal visible en la población.

El problema es que las poblaciones son en realidad heterogéneas en varios sentidos, por lo que los individuos no corren el mismo riesgo de morir ante cada causa, de manera que la muerte resulta selectiva. Es así como las muestras de mortalidad en una población siempre incluirán el componente selectivo y el de la casualidad.

Cohen (op. cit.) postula que la mortalidad fue no selectiva durante mucho tiempo en las sociedades prehistóricas, pero Wood y Milner (1994) opinan que tal afirmación parece basarse en que Cohen piensa que la mayoría de las muertes fueron accidentales. El problema es que los datos, tales como existen hasta ahora, sugieren que la abrumadora mayoría de las muertes en todas las sociedades preindustriales son de naturaleza infecciosa, siendo la desnutrición una frecuente causa contribuyente. Se sabe que las enfermedades infecciosas son altamente selectivas y están relacionadas especialmente con el estado nutricional y con la función inmune.

Cuando en 1992 Wood et al. elaboraron lo que denominaron 'la paradoja osteológica', sugirieron que una serie de factores que incluyen la no estacionalidad, la heterogeneidad oculta, la fragilidad diferencial y la mortalidad selectiva, pueden sesgar la muestra de esqueletos en un cementerio. De esta manera se obtendría una muestra no representativa de la población que alguna vez vivió y por tanto las conclusiones acerca del impacto económico sobre la salud humana no serían confiables. Entonces, para Wood y Milner (op. cit.) la afirmación de Cohen acerca de que la mortalidad es ampliamente casual y no selectiva es tan profunda en sus implicaciones que se debe sostener con pruebas y no solamente darla por hecho. Mas concuerdan con él en cuanto a que la mortalidad tiene elementos fortuitos (casuales) y deterministas (no casuales).

Estos autores plantean estar conscientes de que en ningún estudio se ha demostrado que los accidentes se traduzcan en causas de muerte. Tampoco hay razón para creer que los accidentes sean genuinamente casuales: 'la 
tendencia a los accidentes' es un fenómeno epidemiológico bien establecido. De la misma manera no se puede asumir que las muertes violentas en oposición a aquellas provocadas por enfermedades o alguna de las demás vicisitudes de la vida estén casualmente distribuidas. Por tanto, incluso los accidentes y la violencia pueden ser selectivos de acuerdo a las características de las personas.

Sin embargo, es importante señalar que a medida que los individuos envejecen su fragilidad aumenta, de modo que este azar de muerte no necesariamente declina. La fragilidad en edades avanzadas, es producto de diversos factores que se van acumulando en el transcurso de la vida de los individuos como es el tipo de dieta consumida, el stress al que estaban expuestos y factores genéticos que se expresan en la vejez. De este modo, puede cuestionarse el que el riesgo individual permanezca constante.

En 1984, Cohen et al. señalaron que debido a la transición desde una economía de caza y recolección a una economía agrícola, los individuos se ven sometidos a un alto stress y por ende la supervivencia de ciertos grupos etarios se ve comprometida. Destacan así dos indicadores que implican un deterioro generalizado de la calidad de vida de los primeros agricultores. En 1989, Cohen agrega más datos acerca de este hecho, demostrando que la agricultura y el sedentarismo causaron un aumento de la contaminación medioambiental local, la transmisión de las infecciones persona a persona se hizo más frecuente y la calidad y diversidad de la dieta disminuyeron.

Sin embargo, por otra parte, estos mismos datos pueden interpretarse de otra manera. Sattenspiel y Harpending en 1983 y luego Milner et al. (1989), sugirieron que la primera hipótesis era muy posible pero que el cambio en la edad promedio al morir podría estar reflejando un aumento de la fertilidad mientras que la tasa de mortalidad se mantuvo constante y no hubo un deterioro de la salud general. Si realmente ocurrió este aumento de la fertilidad, podría deberse a que existió una mayor disponibilidad de alimentos para poder destetar a los niños a una edad más temprana, de forma que resultan períodos más cortos de infecundidad producto de la lactancia entre los primeros agricultores. Asimismo, las lesiones óseas causadas por el stress reflejarían un aumento de la habilidad para sobrevivir a las nuevas enfermedades y exigencias medioambientales, o un mejoramiento frente a ciertas causas de muerte imposibles de observar en el estilo de vida anterior.

Es importante destacar que ambas interpretaciones son posibles y que ninguna de las dos es necesariamente más correcta que la otra. Lo más probable es que ambas sean correctas para diferentes períodos y lugares, pues hasta ahora los datos apoyan ambas interpretaciones con la misma fuerza. El paleopatólogo se enfrenta aquí al hecho de que la relación entre la evidencia esqueletal de las patologías y las actividades no es tan directa como para el arqueólogo, pues mientras que los arqueólogos han estado trabajando con la relación artefacto-actividad durante un largo período de tiempo, los intentos 
de estudiar los restos osteológicos en términos de conducta son comparativamente escasos y recientes. En resumen, para poder elegir cual (o cuales) es la mejor de las interpretaciones, es necesario un estricto control del contexto cultural y una profunda comprensión de la fragilidad biológica individual y de la mortalidad selectiva.

La naturaleza de las patologías puede ser difícil de elucidar, debido a que muchas de las enfermedades y/o deficiencias nutricionales actúan siguiendo los mismo procesos lo cual, puede generar resultados semejantes y, por tanto la identificación de un agente causal único no es posible. Con todo ello, se observa que la interpretación de la aparición e incidencia de las patologías en relación al fenómeno del cambio nutricional es complejo, puesto que nutrición y enfermedad se encuentran intrínsecamente relacionadas (Axerold; Roosevelt).

Así como la malnutrición puede disminuir la resistencia de un individuo a la enfermedad, una enfermedad también puede causar malnutrición al incrementar la necesidad del organismo por ciertos nutrientes. Además, una dieta insuficiente y/o desbalanceada afecta la producción de anticuerpos en el organismo, debido a que éste no sólo genera menos anticuerpos sino que, además, presenta una menor cantidad de células B productoras de anticuerpos (Axerold). Por tanto es necesario que el estudio de la salud y la nutrición se realice en forma conjunta, de modo tal que permita distinguir las interpretaciones paradójicas de aquellas que no lo son (Constantinescu \& Alfonso, 1996).

Por tanto, si se estudia el tipo de dieta y se maneja esta información conjuntamente con otros indicadores bioantropológicos, como son las patologías y la edad de muerte, se pueden realizar interpretaciones más amplias y con un menor riesgo de error, pues la nutrición o más bien, el estado nutritivo de los individuos, tiene una fuerte incidencia en la resistencia-sensibilidad a las infecciones (Axerold).

\section{Paleopatología}

El cuerpo humano debe efectuar una serie de tareas muy diferentes durante el transcurso de una vida, sufriendo cambios que se miden en términos de desgaste de tejidos. Es así como los humanos dejan un registro de sus actividades en sus propios huesos. Sin embargo lo anterior no hace necesariamente referencia a prácticas culturales como la deformación craneana intencional o la ablación dental, sino al registro de uso y desgaste dejado sin intención durante el transcurso de las actividades diarias. Este registro está representado por las fracturas, las pérdidas de dientes, las artritis y, quizás también en términos sutiles, por el alineamiento trabecular y por la composición química del hueso.

Sin duda, los esqueletos son la mejor fuente de información sobre las enfermedades antiguas, sin embargo debe considerarse que hay relativamente 
pocas condiciones mórbidas que afectan al esqueleto de manera de dejar cambios visibles en los huesos. La patología ósea puede caracterizarse como un proceso de destrucción ósea, de formación ósea anormal o como una mezcla de ambos. El hecho de realizar alguna actividad habitual u ocupacional impone condiciones de stress prolongado y continuo bajo las cuales se pueden llegar a desarrollar irregularidades en los tejidos óseos y dentales. La notoria deformidad ósea producto de un stress severo y prolongado constituye el marcador de stress ocupacional que puede observarse macroscópicamente. Asimismo, aquellos músculos que tienen una influencia directa sobre la morfología ósea en los lugares que no son superficies articulares, constituyen otro tipo de respuesta. Los marcadores de stress ocupacional, que son básicamente respuestas ante las acciones de tirar y empujar, se manifiestan macroscópicamente cuando un área del hueso se ve afectada por una fuerza o carga que excede el límite de la elasticidad del hueso y el área sometida a stress no vuelve a su forma original.

La importancia de estas 'patologías inducidas por actividad' o 'patologías morfofuncionales' es que no son azarosas por lo que reflejan la naturaleza no azarosa de la actividad en sí. Es verdad que las actividades desarrolladas y las posturas adoptadas durante un día diferirán de las de cualquier otro, pero existen ciertas similitudes. Una y otra vez se repetirán los mismos tipos de actos y posturas, sobre todo si se consideran "correctos" desde un punto de vista cultural, o si son necesarios para sobrevivir. Debe por tanto enfocarse el interés en la conducta habitual, mejor denominada como "patrones de actividad".

Estas huellas que se encuentran en el esqueleto y que nos acercan a la comprensión de los 'modos de vida' de las sociedades pasadas, se conocen como 'indicadores de stress'. El stress es producto de tres sets de factores: las restricciones medioambientales, los sistemas culturales y, la resistencia del huésped (Goodman et al., 1984a). Las restricciones medioambientales incluyen tanto los recursos limitados como los factores de stress, los cuales, si no son 'amortiguados' por la cultura de la cual participan los individuos pueden llegar a generar un incremento en las lesiones patológicas. Así, las condiciones ambientales pueden ser suavizadas por el sistema cultural, sin embargo una cultura puede, por el contrario, aumentar los factores de stress existentes o crear otros nuevos. Luego, si el stress no es adecuadamente paliado por medios extraindividuales (por ende sociales o culturales), su efecto sólo puede ser contrarrestado a través de la resistencia individual. Dicha resistencia varía de acuerdo con la edad y el sexo de los individuos, además de la incidencia de factores genéticos (Goodman et al., 1984a; Huss-Ashmore et al.).

Goodman et al. (op. cit.), clasifican los indicadores de stress bajo tres categorías mayores:

1. indicadores generales de stress acumulativo. 
2. indicadores generales de eventos de stress episódicos.

3. indicadores de stress asociados a enfermedades específicas.

Los indicadores generales acumulativos incluyen los cálculos de mortalidad y crecimiento ya que, bajo condiciones de stress un individuo que se encuentra aún en su etapa de desarrollo puede, ya sea reducir o cesar por completo su crecimiento, debido a que los nutrientes con que cuenta son utilizados para combatir el stress en lugar de permitir el crecimiento, por lo cual este último puede verse disminuido o detenido por completo al menos por un lapso de tiempo. Si la energía consumida decrece, los requerimientos del crecimiento son enfrentados cada vez en forma más deficiente, de modo que tanto el crecimiento longitudinal como el transverso se ven desacelerados (Goodman et al., 1984a; Huss-Ashmore et al.; Smith et al.; Kennedy; Larsen, 1987; Martin et al.; Snyderman).

Los indicadores de stress periódicos o episódicos nos entregan información acerca de la edad en la que ocurrieron los eventos de stress. Existen dos ejemplos muy comunes de este tipo de indicadores: el primero de ellos es conocido como líneas de Harris, que consisten en disrupciones lineales en el crecimiento del hueso (Huss-Ashmore et al.; Wood et al., 1992; Goodman et al, 1984a; Roosevelt; Cook; Perzigian et al.). El segundo indicador es la hipoplasia del esmalte, que se define como una disrupción en la formación de la matriz del esmalte dental que es resultado de un cese en la amelogénesis (formación del esmalte), que provoca una deficiencia en el espesor de éste. Además, como el esmalte una vez formado no puede ser absorbido o remodelado, provee de un registro permanente y, lo que es más, cronológico del episodio de stress ya que, de acuerdo a la distancia en que se encuentre con respecto a la unión esmalte-cemento, se puede determinar la edad en que se presentó dicho evento (Skinner y Goodman; Wood et al., 1992; Goodman et al., 1984a, 1984b; Goodman, 1993; Cohen, 1994; Roosevelt; Lukacs; Cohen y Armelagos, 1984; Cook; Cassidy; Perzigian et al.; Goodman y Armelagos, 1985; Goodman y Rose, 1990).

En el contexto paleopatológico mayor, es necesario ser cuidadoso al efectuar inferencias acerca del estado de salud relativo de la población, basándose en la incidencia de la patología esqueletal. Se tienden a asociar las manifestaciones esqueletales de estados mórbidos con respuestas crónicas a la enfermedad. La paleopatología esqueletal, particularmente las infecciones, tienden a considerarse como evidencia de alta morbilidad, sin embargo también es posible que se trate de lo contrario. La paleopatología esqueletal puede estar indicando una mejor respuesta del huésped frente a la enfermedad, puesto que sobrevivió al estado agudo de ésta mientras que otros individuos afectados pueden haber muerto. Así, la ausencia de la patología esqueletal indicaría muerte frente a condiciones agudas, mientras que la evidencia de patología esqueletal indica que una respuesta inmunológica es lo suficiente- 
mente adecuada como para asegurar la supervivencia del individuo hasta que la enfermedad se hace crónica.

Las patologías morfofuncionales afectan por lo general a los tejidos blandos y al esqueleto, pero es este último el que tiene importancia en un análisis de restos arqueológicos. El contexto arqueológico es extremadamente importante pues da cuenta de la cultura particular a que pertenecen los hallazgos, de las influencias ambientales a las que esta cultura se vio enfrentada y de las condiciones de conservación y recuperación de los restos.

Para estudiar los aspectos paleopatológicos en una población, debemos trabajar con supuestos osteológicos, que de acuerdo a Bridges pueden verificarse mejor en los períodos de grandes cambios de la historia humana como son la transición hacia la agricultura y la llegada de los europeos a América. Es precisamente dentro del primer período en que se inserta esta tesis $y$, hasta ahora, se han definido las siguientes tendencias mundiales en los estudios de patologías de esqueletos y momias de cazadores-recolectores y agricultores (Cohen, 1994):

1. la frecuencia de infecciones crónicas no-específicas encontradas en los esqueletos, es generalmente más alta entre los agricultores que entre los cazadores-recolectores;

2. la frecuencia de infecciones específicas como la yaws y la tuberculosis o infecciones similares a la tuberculosis, generalmente aumentan a medida que los grupos se hacen mayores y aumenta el sedentarismo;

3. la frecuencia de infecciones intestinales y los parásitos aumentan junto al tamaño del grupo y su detección también aumenta al analizar momias o fecas;

4. la frecuencia de la hiperostosis porótica, lesión esqueletal de la anemia infantil, es casi siempre mayor entre los agricultores que entre los cazadores-recolectores;

5. otros signos de desnutrición (crecimiento retardado y osteoporosis en los niños, osteoporosis prematura en los adultos, tamaño reducido de los dientes, etc.) son más comunes entre los agricultores que entre los cazadores-recolectores;

6. la estatura promedio de los individuos adultos medidos declinó a lo largo del Viejo Mundo, desde el Paleolítico hasta el Neolítico;

7. los signos de stress sistemático visibles en los dientes, incluyendo la hipoplasia del esmalte (macroscópica) y las bandas de Wilson (microscópicas), son generalmente más frecuentes y pronunciadas entre los agricultores que entre los cazadores-recolectores.

Asimismo, se considera por lo general y, a menudo sobre la base de los esqueletos del sitio Dickson Mounds — sudeste de Estados Unidos-, que una 
mayor mortalidad o un aumento de población, siguió a la introducción de la agricultura (Bridges, op. cit).

De acuerdo a los estudios realizados hasta la fecha, a través del mundo los grupos agrícolas tienden a presentar más enfermedades infecciosas que los cazadores-recolectores (Bridges, op. cit). También puede haber una declinación en la salud nutricional (como lo indican los niveles más altos de hiperostosis porótica o cribra orbitalia que pueden deberse a una anemia dietaria) (Cohen y Armelagos, 1984). Algunos grupos agrícolas tienen también tasas de crecimiento más lentas. Otros indicadores de salud, como la estatura de los adultos y el dimorfismo sexual muestran más variación y pueden aumentar, disminuir o permanecer iguales (Cohen y Armelagos 1984, Goodman et al. 1984).

Wood et al. (1992) postulan en particular, que el aumento aparente de la patología, asociado a las poblaciones agrícolas tempranas, podría de hecho estar reflejando un mejoramiento de la salud. Sugieren que las poblaciones agrícolas pueden haber estado mejor alimentadas y ser más longevas que sus antecesores cazadores-recolectores, debido a lo cual es más probable que registren stress en sus esqueletos. Según esta interpretación, la frecuencia de la infección (o de otras patologías) no aumenta con la agricultura, sino que es un registro más completo y mejor conservado en los esqueletos de las poblaciones mejor alimentadas y más longevas. Los esqueletos de los cazadores-recolectores están relativamente libres de patologías, no porque estas poblaciones fuesen más saludables, sino porque morían antes que los esqueletos pudieran registrarlas. Sugieren que los promedios de edad bajos al morir y las altas frecuencias de hipoplasia del esmalte de la población posterior totalmente agrícola, que antes eran interpretados como un registro de disminución de la calidad de la salud y de la longevidad (Goodman et al. 1984) deben ser indicativos de un aumento de la fertilidad y de privilegio biológico.

Por tanto se puede argumentar que de acuerdo a Wood et al., la alimentación y la salud mejoraron con la agricultura y el sedentarismo en las poblaciones prehistóricas, o también que la supervivencia era significativamente mayor entre los grupos agrícolas tempranos que entre los cazadores-recolectores. Los datos arqueológicos sugieren que los cazadores-recolectores fueron adoptando la agricultura a medida que abandonaban las estrategias económicas que alguna vez fueron importantes. En general los cazadores-recolectores deben haber tenido un mejor conocimiento nutricional, así como un menor conocimiento de las infecciones que los agricultores y, normalmente deberían haber sido los más adaptables, incluso pese a las presiones de la movilidad que, en sí, debe haber actuado en su contra.

Sin embargo, Cohen opina que tal vez, ni la supervivencia ni la fertilidad deben haber cambiado mucho durante la transición hacia la agricultura, a menos que los dos cambios se hayan efectuado en direcciones opuestas. Generalmente los mejores cálculos sugieren que la tasa de crecimiento para 
nuestra especie en conjunto, aumentó de un promedio de $0.01 \%$ anual antes de la adopción de la agricultura, a un $0.1 \%$ después de la adopción de ésta. Incluso si se asume que todo el aceleramiento resultó del aumento de la supervivencia sin que el aumento de la fertilidad haya contribuido, simplemente no hubo un mejoramiento suficiente de la supervivencia promedio, como para considerar que el aumento de la patología sea visible (Cohen 1994).

Desde el punto de vista de la presencia de patologías morfofuncionales en los esqueletos, el estudio de Bridges demuestra que en general los agricultores Mississippi tienen las diáfisis de sus huesos largos más gruesas y más fuertes que las de los cazadores-recolectores arcaicos. Lo anterior sugiere que estaban abarcando actividades más forzadas pues en esta región del sudeste de Estados Unidos, la agricultura requirió entonces mayor fuerza ósea que lo que requerían la caza y la recolección. Existen distintos patrones de cambio esqueletal en las inserciones musculares de hombres y mujeres, los que sugieren un cambio en la división de las labores habituales. Dicho cambio coincide con el cambio hacia la agricultura del maíz. La mayor difusión de los aumentos de fuerza en las mujeres sugieren un aumento en la variedad de tareas; los hombres, que muestran diferencias menores, pueden haber sido menos afectados por la introducción del maíz.

Se puede inferir que el cambio en la subsistencia desde una economía cazadora-recolectora a una economía agrícola también está ligado al cambio en la división sexual del trabajo. Las mujeres asumieron una mayor proporción de las actividades de subsistencia asociadas con la agricultura y esto se ve reflejado en los cambios en las diáfisis de sus huesos largos, que están más difundidos que entre los hombres. Estos hallazgos son congruentes con la evidencia derivada de grupos agrícolas modernos y de los registros históricos de las sociedades indígenas del sudeste de Estados Unidos (Bridges, op. cit.).

Frente a este punto, Wood y Milner (1994) destacan la importancia del 'contexto cultural' en la interpretación de los esqueletos provenientes de cementerios. Señalan que el total de los cambios en las dietas y en los estilos de vida relacionados con una dependencia creciente en los cultivos, tomó en realidad un período mucho más largo que los pocos siglos en que fue usado el cementerio Dickson Mounds, y el maíz fue una incorporación tardía a las dietas de entonces, basadas en gran parte en diversos cultígenos. Así, a pesar de todos los trabajos en Dickson Mounds, hasta ahora no disponemos de todo el espectro de cambios esqueletales producidos por el cambio desde la cazarecolección a la agricultura.

En nuestro país, esta línea ha comenzado a surgir tímidamente, aplicándose en forma específica al ámbito forense desde 1985 (Paredes, com. pers.). En el caso de la investigación arqueológica, los trabajos son aún más recientes, comenzando a ser publicados a comienzos de la década de los '90. La reconstrucción de un 'modo de vida' pasado tiene sin embargo una importan- 
cia invaluable, si se logra disponer de la información necesaria y ésta se interpreta adecuadamente.

\section{DISCUSIÓN}

La interdisciplinariedad apela a la ampliación de la comprensión de un fenómeno por medio de su estudio conjunto por parte de distintos especialistas. La interdisciplinariedad enfrenta entonces distintas perspectivas, proveyendo de una visión más integral, más acabada de un fenómeno, al desafiar la comprensión del mismo. Así dos o más disciplinas contrastan, cotejan, corrigen, complementan, mejoran y amplían su conocimiento.

El concepto de interdisciplinariedad es tomado por el realismo constructivo desde su punto de vista práctico: siempre ha sido necesario en ciencia un objeto de estudio y un método para estudiarlo, sin embargo esto constituye un círculo pues para escoger el método adecuado debería conocerse su objeto de antemano, pero para conocer el objeto, es necesario un método. Ante este problema fundamental en la ciencia el Realismo Constructivo pide no negarlo --incluirlo dentro de la interpretación científica-, pero más que eso, pide comenzar un profundo proceso de reflexión en torno a la ciencia (Wallner).

De hecho, este proceso de reflexión ya ha comenzado, pues tal como plantea Arnold, es imposible negar que esta última década ha visto fuertemente criticada la orientación investigativa tradicional, basada en lo analítico y lo causal. Ha surgido una nueva propuesta epistemológica que apela a un conocimiento dinámico que emerge del contexto en que se desarrolla la observación.

En el caso particular de las ciencias sociales, la orientación sistémica propone un nuevo tipo de enfoque basado en el concepto de que los sistemas sociales son horizontes compartidos de sentido. Su función es reducir la contingencia y operar como 'estructuras estructuradas estructurantes'. Además, no debe ignorarse que en el ámbito de las ciencias sociales, observador, método y objeto se afectan mutuamente al ser contemporáneos.

La interpretación científica pasa a ser una construcción cultural, una pauta configurada, un orden de experiencias ajenas hechas propias, una comparación que construye mapas, modelos, representaciones. La objetividad se traslada, ya no se comprende como la verdad alcanzable, sino como dominios de significatividades. La objetividad que interesa es aquella coproducida y sostenida por los observados (Arnold).

Para desentrañar las formas del ver y del leer cotidiano, encontrar los esquemas de distinción compartidos, se postula la observación de segundo orden: observar cómo observan los demás. Y mientras más observaciones se hagan, se llegará a más explicaciones, que al ser tomadas en conjunto - interdisciplinariamente — constituyen la explicación más probable. 
En el acápite anterior, se han planteado los principales postulados de la bioantropología en torno a la problemática de la reconstrucción de los 'modos de vida'. Se hace evidente que la comprensión de los fenómenos paleopatológicos es un proceso que implica interpretar datos de carácter biológico, de acuerdo a diferentes tipos de indicadores. Según el tipo de indicador seleccionado, es posible aproximarse a aspectos tales como calidad de vida, actividades realizadas, estado de salud y/o nutrición entre otros. Utilizados en conjunto, permiten acceder a la comprensión del 'modo de vida' de las poblaciones pasadas. Sin embargo, al igual que todo en arqueología, se trata de una interpretación y por ello es una constante controversia decidir de qué modo ésta debe realizarse, es decir, definir cuáles son los fundamentos que la hacen sólida (Constantinescu \& Alfonso, 1996).

De acuerdo a la bioantropología, dentro del contexto paleopatológico mayor, se debe tener cuidado al inferir el estado de salud relativo de la población, estimándolo en base a la incidencia de la patología esqueletal. Como se ha señalado, en general, se asocian las manifestaciones esqueletales de estados mórbidos con respuestas crónicas a la enfermedad. Sin embargo, también puede tratarse de lo contrario, de modo que es posible que la ausencia de patología esqueletal indique muerte frente a condiciones agudas, mientras que la evidencia de patología implica supervivencia - vale decir, una respuesta inmunológica adecuada-.

Aún así, para que esta lectura sea adecuada, es necesario considerar la edad de muerte de los individuos. Esto debido a que, si se trata de un individuo adulto, la ausencia de patología esqueletal no necesariamente indica la presencia de un proceso mórbido de rápido avance, sino probablemente la de una condición propia del envejecimiento, conocida como apoptosis (Alfonso).

El desafío que plantea la lectura bioantropológica es casi insoluble si sólo se trabaja con los datos biológicos. Para la arqueología, la interpretación más lógica de la evidencia paleopatológica en cuanto al estado de salud de las poblaciones en transición hacia la agricultura, es la postura de Wood $e t$ al.

Está claro que las sociedades agrarias tempranas están viviendo un proceso de cambio, en que hay un trabajo hacia la adopción de la agricultura, pero con un fuerte énfasis en la caza-recolección. No se abandona súbitamente una estrategia económica para abrazar una diferente, sino que hay un desarrollo paulatino de nuevas técnicas y se sigue dependiendo fuertemente de la cazarecolección. De hecho, esta estrategia nunca es abandonada completamente, siempre complementará una dieta agrícola, aportando a la gama de recursos cultivados disponibles, otros tipos de recursos silvestres, no domesticados, que por lo general son estacionales.

¿Qué supuestos pueden entonces desprenderse de la reconstrucción de 'modos de vida' bioantropológica para el período de adopción de la agricultura? Constantinescu y Alfonso proponen que según esta lectura, aparentemente la mayor parte de las poblaciones prehistóricas que se encontraban 
practicando una agricultura intensiva sufría de una malnutrición crónica a lo largo de su vida, especialmente durante la infancia. La causa de este stress nutricional parece encontrarse en la pobreza de nutrientes presentes en los cultivos estables.

Según las evidencias obtenidas, el cambio en la subsistencia prehistórica se mueve hacia la explotación de recursos con mayor capacidad de soporte y no hacia alimentos de mayor valor nutricional. Es decir, el cambio en el "modo de vida' y por tanto en la dieta no conllevó una mejora en las condiciones de salud de los individuos sino, al parecer, todo lo contrario (Alfonso).

Pero los datos bioantropológicos tratados aisladamente, sólo logran proporcionar esta visión. Es necesario recurrir a fuentes distintas de interpretación para ampliar su comprensión. Por ejemplo, con respecto al tema de la adopción de la agricultura, los estudios etnográficos muestran que, a diferencia de los cazadores-recolectores, los grupos agrícolas y los pastores consumen un espectro más limitado de recursos alimenticios. Ellos intentan evitar el riesgo que representan las hambrunas a través del cultivo intensivo de los recursos que son culturalmente favorecidos, o que se consideran mejores. En cambio, los cazadores-recolectores evitan el riesgo de las hambrunas explotando una amplia variedad de alimentos (Constantinescu \& Alfonso, 1996).

Aunque durante los últimos años, la bioantropología ha ido ampliando su ámbito interpretativo, prestando atención a otros campos de estudio, este esfuerzo ha sido insuficiente. Así, por ejemplo, al trabajar los esqueletos de un sitio arqueológico, el bioantropólogo sólo hace una breve referencia al período y cultura a que se adscribe el sitio y, a algunos datos de interés particulares. Continúa luego con la interpretación de la evidencia ósea en base a estas generalidades.

Los datos bioantropológicos así tratados, inducen negativamente las interpretaciones posteriores. Así por ejemplo, la arqueología tiende a enfatizar las dicotomías existentes entre los distintos sistemas económicos. Y un arqueólogo que trabaja con este tipo de supuestos bioantropológicos, integrándolos a los resultados de los estudios del contexto arqueológico, ve reforzarse las dicotomías. De tal manera que dichos sistemas suelen considerarse prácticamente excluyentes. Sin embargo, los datos que aporta la bioantropología permiten considerar matices, estados intermedios, profundizando aspectos que otros tipos de análisis no han precisado.

Es evidente que el análisis y la comprensión de la evidencia paleopatológica son complejos, razón por la cual se deben utilizar múltiples indicadores (Goodman, 1993), entre los que se incluye el registro arqueológico. Es necesario tener presente que, aunque las muestras esqueletales tienen limitaciones para el estudio de las poblaciones pasadas (como cualquier tipo de registro arqueológico) se pueden obtener importantes conclusiones si se realizan los controles adecuados (Goodman et al., 1993; Larsen, 1987). 
Por tanto, no debe olvidarse que los grupos humanos son muy diversos, sus elecciones son por tanto distintas y, lo mismo sucede con el medioambiente que ellos habitan: cada grupo representa algo único en su 'modo de vida', en su cultura y es por lo tanto un constante desafío lograr su comprensión (Constantinescu \& Alfonso, 1996). A pesar de esta gran diversidad, las respuesta óseas frente al stress son limitadas, lo que remite a patrones de actividad definidos, que analizados en conjunto con todo el registro arqueológico, permiten llegar a la individualidad de cada cultura.

Por otra parte, es necesario indicar que es de suma importancia que todos los indicadores bioantropológicos de stress, sea cual sea su clasificación, sean analizados en forma conjunta. Deben establecerse interrelaciones entre ellos para poder llegar a comprender qué es lo que estaba sucediendo a nivel fisiológico con el individuo al momento de morir. Es decir, qué afecciones estaba sufriendo y, cuáles lo habían afectado en el transcurso de su vida.

Lo más probable es que determinar la etiología precisa de éstos sea un trabajo arduo, pero posible. Si se utiliza tanto la información arqueológica, como la arqueobotánica y la arqueobiológica con el fin de acceder a los diversos factores a que se encontraba sometida la población y cuales eran sus respuestas culturales, se podrá al fin comprender aquellas que presentan un carácter individual.

Así, los estudios paleopatológicos son capaces de establecer el proceso por el cual las culturas pueden actuar como causa o respuesta a los factores de stress y, por lo tanto enriquecer nuestro conocimiento sobre el hombre (Constantinescu \& Alfonso, 1996).

Aquí es donde cabe la propuesta del 'extrañamiento'. Los supuestos bioantropológicos en torno a la reconstrucción de 'modos de vida' no operan por sí solos. Necesitan de un marco teórico mayor, donde al insertarse formen parte de las variables a considerar.

De manera que se ha propuesto que en arqueología, 'modo de vida' sea comprendido como los patrones conductuales definidos por una cultura particular, con que sus miembros deben operar en ambiente físico determinado. Así, resulta natural utilizar los supuestos y métodos bioantropológicos para tratar este problema en arqueología, interpretando los resultados dentro del registro arqueológico. Y, particularmente en el caso de los sitios tardíos e históricos, la evidencia etnohistórica y etnográfica también debe incluirse en las interpretaciones.

Se rescata entonces la propuesta de Borrero de incorporar al registro arqueológico los restos óseos humanos: hay un elemento más que ahora debe ser considerado. En efecto, la relación entre bioantropología y arqueología es evidente al estudiar las patologías morfofuncionales. Son éstas las que se pueden asociar a artefactos, ecofactos y rasgos. Sin olvidar que estas relaciones son complementadas por los indicadores de dieta, demografía y distancias biológicas. 
Se trata de cambiar la perspectiva, de analizar los datos biológicos desde un punto de vista cultural. Es el otro lado de la propuesta de Clarke: la reconstrucción de 'modos de vida' permite encontrar la acción fósil, descubrir la secuencia de actividades petrificadas que equivalen a un artefacto, definir la pauta completa de comportamiento que se parangona con un conjunto de artefactos. Porque la cultura también se refleja en el organismo humano.

\section{CONCLUSIONES}

Ante la evidente e histórica separación entre bioantropología y arqueología, se ha propuesto un puente para su encuentro. Este se construye 'extrañando' el cuerpo teórico de supuestos bioantropológicos referentes a la reconstrucción de los 'modos de vida', al ámbito de la arqueología.

Al efectuar esta operación, queda en inmediata evidencia la discrepancia en torno a lo que cada disciplina entiende por 'modo de vida'. La definición arqueológica es precisa y tradicionalmente se limita a los cazadores-recolectores, mientras que la definición bioantropológica es literal, global, se aplica a cualquier grupo humano sin mayor discusión.

Por tanto se propone una nueva definición de 'modo de vida': son los patrones conductuales definidos por una cultura particular, con que sus miembros deben operar en un medio físico determinado. Esta definición evita enfáticamente proponer que la finalidad de una cultura al adoptar un 'modo de vida' determinado sea la adaptación al medioambiente, pues considera que cada cultura define sus elecciones de acuerdo a sus propios parámetros, sin estar determinada por el medio y, porque adaptación es un concepto que no tiene un claro referente en el registro arqueológico.

Se ha establecido la relación entre la reconstrucción de 'modos de vida' bioantropológica y la ecología cultural, marco teórico propio de la arqueología. Al analizar cómo opera cada disciplina por separado, teniendo en mente que su meta es la reconstrucción de los 'modos de vida', en el sentido de la nueva definición propuesta, quedan en evidencia los sesgos que introduce la aplicación independiente de cada uno.

Por un lado, la ecología cultural difícilmente podría abarcar la definición que se propone para 'modo de vida'. De acuerdo a lo que se puede desprender de su marco teórico, los 'modos de vida' deberían reducirse a la expresión concreta y singular de los modos de producción, modelo teórico que deja fuera de registro aquellas actividades que no son actividades de subsistencia. Así, la ecología cultural y los enfoques posteriores que de ella se derivan, no proporcionan los elementos necesarios para trabajar íntegramente el problema planteado.

La bioantropología por su parte, ha estado desarrollando dos líneas de trabajo paralelas, la paleodemografía y la paleopatología, que en conjunto han 
constituido la base de esta disciplina en la reconstrucción de los 'modos de vida'. Esta línea conlleva a la interpretación de datos de carácter biológico con el fin de acercarse a la comprensión del 'modo de vida' de las poblaciones pasadas. El análisis de esta evidencia es complejo, razón por la cual se deben utilizar múltiples indicadores, entre los que se incluye el registro arqueológico. Sin embargo, la incorporación de este registro es muy reciente, y sólo se han tomado los aspectos esenciales que definen los sitios arqueológicos.

Para integrar estos dos enfoques, se rescata la propuesta de Borrero: los restos óseos humanos también forman parte del registro arqueológico. Por tanto se desprende que, si bien en el presente artículo se ha analizado la relación entre reconstrucción de 'modos de vida' bioantropológica y ecología cultural, puede establecerse esta relación con cualquier enfoque teórico interpretativo arqueológico. La nueva definición de 'modo de vida' pretende ser aplicable a todos los ámbitos teóricos arqueológicos.

Es evidente hoy, la necesidad de trabajar interdisciplinariamente. Así, 'extrañar' la reconstrucción bioantropológica de 'modos de vida' a la arqueología es un camino para unir ambas disciplinas, que debe ser explorado. Y para esto, hay que cambiar el enfoque clásico: dejar de analizar los datos biológicos sólo desde una perspectiva biológica y comenzar a hacerlo también desde un punto de vista cultural. Será entonces posible proporcionar una respuesta a Clarke: definir qué secuencias de actividades pasadas se parangonan con los artefactos, los ecofactos y los rasgos, al interpretar todos estos datos como parte de un mismo registro.

\section{BIBLIOGRAFÍA}

Alfonso, M., Uso arqueológico de la paleopatología oral. Dieta y subsistencia en una población alfarera de Chile Central: Los Coiles I36 [Santiago], práctica profesional. Depto. de Antropología, U. de Chile (inédito), 1996.

Allison, M. "Paleopathology in Peruvian and Chilean populations". Paleopathology at the Origins of Agriculture, M. Cohen y G. Armelagos (eds.), [Orlando], Academic Press Inc. Ltd., 1984, pp. 515-529.

Arnold, M., "Investigación sistémica: alcances y proposiciones". Estudios Sociales [Santiago], $\mathrm{N}^{\circ} 74, \mathrm{Vol} .4,1992$, pp. 97-118.

Axerold, A., "Nutrición en relación con la inmunidad". Nutrición en la Salud y en la Enfermedad. Conocimientos Actuales, R. Goodhart y M. Shills (eds.) [Barcelona], Ed. Salvat, 1987, pp. 535-547.

Borrero, L., "Historia reciente de la arqueología patagónica". Ruma [Buenos Aires], año XXII. I995, pp. 151-176.

Bridges, P." "Changes in activities with the shift to agriculture in the Southeastern United States". Current Anthropology [Utrecht], v. 30, No 3. 1989, 382-394.

Byers, S., "On stress and stature in the osteological paradox". Current Anthropology [Utrecht], v. $35, \mathrm{~N}^{\circ} 3,1994$.

Cassidy, C. "Skeletal evidence for prehistoric subsistence adaptation in Central Ohio River". Paleopathology at the Origins of Agriculture, M. Cohen y G. Armelagos (eds.) [Orlando], Academic Press Inc. Ltd., 1984, pp. 307-345. 
Clarke, D., Arqueología Analítica ( $2^{\text {a }}$ ed.) [Barcelona], Ediciones Bellaterra S.A., 1978.

Cohen, M., "Paleopathology at the origins of agriculture". Paleopathology at the Origins of Agriculture, M. Cohen y G. Armelagos (eds.) [Orlando], Academic Press Inc. Ltd., 1984. pp. 585-600.

Cohen, M. y G. Armelagos, Paleopathology at the Origins of Agriculture [Orlando], Academic Press Inc. Ltd., 1984.

Cohen, M., Health at the rise of civilization [New Heaven], Yale University Press, 1989.

, "The osteological paradox reconsidered". Current Anthropology [Utrecht], v. 15, $\mathrm{N}^{\circ}$ 5, 1994. pp. 629-637.

Cook, D., "Subsistence and health in the Lower Illinois Valley: osteological evidence". Paleopathology at the Origins of Agriculture, M. Cohen y G. Armelagos (eds.) [Orlando], Academic Press Inc. Ltd., 1984, pp. 235-269.

Constantinescu, F. y J. C. Hagn, "Aspectos bioarqueológicos del cementerio RML 004 'El Valle-Chicauma', período Agroalfarero Tardío, Zona Central de Chile". Actas del XlII Congreso Nacional de Arqueología Chilena [Antofagasta], 1994.

_- "Bioarqueología y reconstrucción del modo de vida en un cementerio de la Cultura Aconcagua". Actas del II Congreso de Antropología Chilena [Valdivia], 1995, pp. 98110.

Constantinescu, F. y M. Alfonso, "Lectura bioantropológica: desafío arqueológico", en: Revista Chilena de Bioantropología, [Santiago], $\mathrm{n}^{\circ}$ 1, publicación electrónica de la Facultad de Ciencias Sociales, U. de Chile, 1996.

Goodman, A. et al.. "Indicators of stress from bone and teeth". Paleopathology at the Origins of Agriculture, M. Cohen y G. Armelagos (eds.) [Orlando], Academic Press Inc. Ltd., 1984a, pp. 13-49.

Goodman, A. et al., "Health changes at Dickson Mounds, Illinois (A.D. 950-1300)". Paleopathology at the Origins of Agriculture, M. Cohen y G. Armelagos (eds.) [Orlando], Academic Press Inc. Ltd., 1984b, pp. 271-305.

Goodman, A., "On the interpretation of health from skeletal remains". Current Anthropology, v. $34, n^{\circ} 3,1993, p p .281-288$.

Goodman A. y G. Armelagos., "The chronological distribution of enamel hypoplasia in human permanent incisor and canine teeth". Archs. Oral Biology [Great Britain], vol. 30, $\mathrm{N}^{\circ} 6,1985$, pp. 503-507.

Goodman A. y J. Rose, "Assessement of systemic physiological perturbations from dental enamel hypoplasias and associated histiological structures". Year Book of Physical Anthropology, Willey-Liss Inc., No 33, 1990, pp. 59- I 10.

Guillén, S., The Chinchorro culture: mummies and crania in the reconstruction of preceramic coastal adaptation in the south-central Andes [Michigan], tesis para optar al grado de Doctor en Filosofía, Universidad de Michigan, 1992.

Huss-Ashmore, R. et al., "Nutritional inference from paleopathology". Advances in Archaeological Method and Theory, M. Schiffer (ed.) [New York], Academic Press, Vol. 5, 1982, pp. $395-474$.

Iscan, M. y K. Kennedy, Reconstruction of life from the skeleton [New York], Alan Liss, 1989.

Jackes, M., "On paradox and osteology". Current Anthropology [Utrecht], v. 34, No 3, 1993.

Kennedy, K., "Growth, nutrition and pathology in changing paleodemographic settings in South Asia". Paleopathology at the Origins of Agriculture, M. Cohen y G. Armelagos (eds.) [Orlando], Academic Press Inc. Ltd., 1984, pp. 169-192.

Larsen, C., "Health and disease in prehistoric Georgia: the transition to agriculture". Paleopathology at the Origins of Agriculture, M. Cohen y G. Armelagos (eds.) [Orlando], Academic Press Inc. Ltd., 1984, pp. 367-392.

Larsen, C., "Bioarchaeological interpretations of subsistence economy and behavior from 
human skeletal remains". Advances in Archatological Method and Theory, M. Schiffer (ed.) [New York], Academic Press, Vol.10, 1987, pp. 339-445.

Lee y De Vore, Man the hunter [New York], Aldine Publishing Company, 1968.

Lukacs, J., "Dental paleopathology: methods for reconstructing dietary patterns". Reconstruction of Life from the Skeleton, M. Iscan y K. Kennedy (eds.) [New York], Alan R. Liss Inc., 1989, pp. 261-282.

Martin, D. et cl., "The effects of sociocconomic change in prehistoric Africa: Sudanese Nubia as a case study", M. Cohen y G. Armelagos (eds.) [Orlando], Academic Press Inc. Ltd.. 1984 , pp. 193-214.

Merbs, C., Patterns of activity induced pathology in a Canadian Inuit population [Ottawa]. Mercury Series, paper N 119 . National Museum of Man, 1983.

Milner, G. et al., "Pattern matching of age at death distributions in paleodemographic analysis". American Journal of Physical Anthropology [Philadelphia|, No 80, 1989.

Ortner, D. y W. Putchar, Identification of pathological conditions in human skeletal remains, [Washington], Smithsonian Institution Press, 1981.

Palkovich, A., "Agriculture, marginal environments, and nutritional stress in the prehistoric South West", en: Paleopathology at the Origins of Agriculture. M. Cohen y G. Armelagos (eds.) [Orlando], Academic Press Inc. Ltd., 1984. pp. 425-438.

Perzigian A. et al., "Prehistoric health in the Ohio River". Paleopathology at the Origins of Agriculture, M. Cohen y G. Armelagos (eds.) [Orlando]. Academic Press lnc. Ltd., 1984. pp. 347-366.

Quiroz, D. et al., Estrategias adaptativas en ecosistemas culturale's insulares: el caso de Isla Mocha [Santiago], informe final proyecto FONDECYT N ${ }^{\circ}$ 92- I 129, 1995.

Roosevelt, A., "Population, health and the evolution of subsistence: conclusions from the conference". Paleopathology at the Origins of Agriculture. M. Cohen y G. Armelagos (eds.) |Orlandol, Academic Press Inc. Ltd., 1984, pp. 559-583.

Rose, J. "Paleopathology and the origins of maize agriculture in the Lower Mississippi Valley and Candoan cultures areas". Paleopathology at the Origins of Agriculture, M. Cohen y G. Armelagos (eds.) |Orlandol, Academic Press Inc. Ltd., 1984, pp. 393-424.

Sackett, J., "Isochrestism and style: a clarification". Journal of Anthropological Archaeology $\mathrm{N}^{\circ} 5,1986$, pp. $266-277$.

Sattenspiel, L. y C. Harpending, "Stable populations and skeletal age". Anerican Antiquity: [Londres], $n^{\circ} 48,1983$.

Sharer, R. y W. Ashmore, Fundamentals of Archaeology [Menlo Park ], The Benjamin/Cummings Publishing Company Inc., 1979.

Skinner, M. y A. Goodman, "Anthropological uses of developmental defects of enamel". Skeletal Biology of Past People: Research and Methods [New York] Willey-Liss Inc.. 1992, pp. 153-174.

Smith, P. "t al., "Archaeological and skeletal evidence for dietary change during the Late Pleistocene/Early Holocene in the Levant". Puleopathology at the Origins of Agriculure. M. Cohen y G. Armelagos (eds.) |Orlando]. Academic Press Inc. Ltd., 1984, pp. 101-136.

Snyderman, S., "Nutrición en la infancia y adolescencia". Nutrición en la Salud y la Enfermedad [Madrid], R. Goodhart y M. Shills (eds.), Salvat, 1987.

Standen, V., El cementerio Morro-1: nuevas evidencias de la tradición funeraria Chichorro (periodo arcaico, norte de Chile) [Lima]. tesis para optar al grado de Maestría en Arqueología, Pontificia Universidad Católica del Perú, 1991

Steele, G. y C. Bramblett. The anatomy and biology of the human skeleton [Texas], Texas A \& M University Press, 1988.

Steward, J., The ory of c ulture change |Urbanal. University of Iltinois Press, 1955.

Wallner, F., "Sobre la realidad efectiva (W) y la realidad (R)". Ocho lecciones sobre realismo contrutivo [Valparaíso], Ediciones Universitarias de Valparaíso, 1994. 
Wood, J. et al., "The osteological paradox: problems of inferring prehistoric health fron skeletal samples". Current Anthropology [Utrecht], v. 13, No 4, 1992, pp. 343-370.

Wood, J. y G. Milner, "Reply", "The osteological paradox reconsidered", M. Cohen. Current Anthropology [Utrecht], v. 15, No 5, 1994, pp. 629-637. 